\title{
Revisit of Semi-Implicit Schemes for Phase-Field Equations
}

\author{
Tao Tang $1,2, *$ \\ 1 Division of Science and Technology, BNU-HKBU United International College, \\ Zhuhai, Guangdong 519087, China \\ 2 SUSTech International Center for Mathematics, Southern University of Science and \\ Technology, Shenzhen, Guangdong 518055, China \\ Received 8 May 2020; Accepted (in revised version) 19 July 2020 \\ Dedicated to Professor Weiyi Su on the occasion of her 80th birthday
}

\begin{abstract}
It is a very common practice to use semi-implicit schemes in various computations, which treat selected linear terms implicitly and the nonlinear terms explicitly. For phase-field equations, the principal elliptic operator is treated implicitly to reduce the associated stability constraints while the nonlinear terms are still treated explicitly to avoid the expensive process of solving nonlinear equations at each time step. However, very few recent numerical analysis is relevant to semi-implicit schemes, while "stabilized" schemes have become very popular. In this work, we will consider semiimplicit schemes for the Allen-Cahn equation with general potential function. It will be demonstrated that the maximum principle is valid and the energy stability also holds for the numerical solutions. This paper extends the result of Tang \& Yang (J. Comput. Math., 34(5) (2016), pp. 471-481), which studies the semi-implicit scheme for the Allen-Cahn equation with polynomial potentials.
\end{abstract}

Key Words: Semi-implicit, phased-field equation, energy dissipation, maximum principle.

AMS Subject Classifications: 65M06, 65M12

\section{Introduction}

There has been tremendous interests in developing energy-dispassion numerical methods for phase-field models starting from earlier numerical works $[1,6,12]$. To make sure a numerical scheme satisfies the nonlinear energy stability, there are basically three class of approaches. For ease of exposition, we consider the simplest phase-field model, i.e.,

*Corresponding author. Email address: ttang@uic.edu.cn (T. Tang) 
the Allen-Cahn equation with initial condition:

$$
\begin{array}{ll}
\frac{\partial \phi}{\partial t}=\varepsilon^{2} \Delta \phi-f(\phi) & \text { in } \Omega \times(0, T], \\
\phi(x, 0)=\phi_{0}(x) & \text { in } \Omega,
\end{array}
$$

where $\varepsilon>0$ is the interface width parameter, $f(\phi)=F^{\prime}(\phi)$, where $F$ is a smooth function.

The corresponding energy is defined as

$$
E(\phi)=: \int_{\Omega}\left(\frac{\varepsilon^{2}}{2}|\nabla \phi|^{2}+F(\phi)\right) d x
$$

Energy stability means that

$$
E(\phi(\cdot, t)) \leq E(\phi(\cdot, s)), \quad \forall t>s .
$$

The first class of energy stable scheme is the Eyre's convex splitting method [6], which yields a nonlinear semi-implicit scheme:

$$
\frac{\phi^{n+1}-\phi^{n}}{\Delta t}=\varepsilon^{2} \Delta \phi^{n+1}-T_{1}\left(\phi^{n+1}\right)-T_{2}\left(\phi^{n}\right),
$$

where $T_{1}$ and $T_{2}$ are some convex functionals satisfying $T_{1}+T_{2}=f$. This is also referred as partially implicit scheme for phase-field modeling by [13].

The second class of energy stable scheme is to add some extra terms so that the resulting scheme satisfies energy non-increasing property; these schemes are called "stabilized" approach. There have been quite large size of papers in this direction in the past 15 years, see the review articles $[9,10]$ and references therein.

The third class is the direct fully implicit scheme, see, e.g., $[5,8,13]$. In particular, it is demonstrated in [13] that a first-order fully implicit scheme for the Allen-Cahn model can be devised so that the maximum principle is valid on the discrete level and, furthermore, the linearized discretized system can be effectively preconditioned using discrete Poisson operators.

It is noted by [6] that an unconditionally energy-stable scheme, such that backward Euler scheme, is not necessarily better than a conditionally energy stable scheme when the time step size is not small enough. In other words, if larger time steps are needed then the first and second classes schemes are useful. However, it is argued in [13] that most implicit schemes are energy-stable if the time-step size is sufficiently small. Moreover, it is noted that a convex splitting scheme or a "stabilized" approach can be equivalent to some fully implicit scheme with a different time scaling and thus it may lack numerical accuracy.

It is obvious that the partially implicit scheme and the fully implicit scheme all require some iteration techniques, and are less effective than the explicit scheme or semi-implicit 
scheme. In this work, we wish to study the semi-implicit energy-stable scheme for the Allen-Cahn equation:

$$
\frac{\phi_{j}^{n+1}-\phi_{j}^{n}}{\Delta t}=\varepsilon^{2} \nabla_{+} \nabla_{-} \phi_{j}^{n+1}-f\left(\phi_{j}^{n}\right), \quad 1 \leq j \leq J,
$$

where $\Delta t$ and $\Delta x$ are time step and mesh size in space, respectively, and

$$
\nabla_{+} u_{j}=\frac{u_{j+1}-u_{j}}{\Delta x}, \quad \nabla_{-} u_{j}=\frac{u_{j}-u_{j-1}}{\Delta x} .
$$

It will be demonstrated that the maximum principle is valid for (1.5), and the energy stability also holds for the numerical solutions.

The paper is organized as follows. In Section 2, we will prove the maximum principe and the $L^{1}$-stability for the numerical solutions of semi-implicit scheme (1.5). The energy stability will be established in Section 3. Some possible extensions will be discussed in the final section.

\section{Maximum principle}

It is known that a maximum principle is satisfied for the Allen-Cahn equation (1.1a), see, e.g., [7,11]. Below we will provide a discrete counterpart using a monotone scheme arguments.

Theorem 2.1. Consider the semi-implicit scheme (1.5) with periodic boundary conditions. Assume $\gamma_{+}>\gamma_{-}$are two constants

- If the nontrival function $f \in C^{1}\left(\gamma_{1}, \gamma_{+}\right)$satisfies

$$
f\left(\gamma_{-}\right)=f\left(\gamma_{+}\right)=0, \quad \gamma_{-} \leq \phi_{0} \leq \gamma_{+},
$$

- and if the time step $\Delta t$ satisfies

$$
\Delta t \max _{\gamma_{-} \leq u \leq \gamma_{+}} f^{\prime}(u) \leq 1
$$

then

$$
\gamma_{-} \leq \phi_{j}^{n} \leq \gamma_{+}, \quad \forall 1 \leq j \leq J, \quad n \geq 0 .
$$

Proof. We use mathematical induction to prove the result. The result is obvious at $n=0$. Assume (2.3) is true at level $n$. It follows from (1.5) that

$$
\phi_{j}^{n+1}=\phi_{j}^{n}+\lambda\left(\phi_{j+1}^{n+1}-2 \phi_{j}^{n+1}+\phi_{j-1}^{n+1}\right)-\Delta t f\left(\phi_{j}^{n}\right),
$$


where $\lambda=: \epsilon^{2} \Delta t / \Delta x^{2}$. Consequently, we have

$$
\begin{aligned}
(1+2 \lambda) \phi_{j}^{n+1} & =\phi_{j}^{n}+\lambda \phi_{j+1}^{n+1}+\lambda \phi_{j-1}^{n+1}-\Delta t f\left(\phi_{j}^{n}\right) \\
& =: G\left(\phi_{j}^{n}, \phi_{j+1}^{n+1}, \phi_{j-1}^{n+1}\right) .
\end{aligned}
$$

Note that

$$
\frac{\partial G}{\partial \phi_{j}^{n}}=1-\Delta t f^{\prime}\left(\phi_{j}^{n}\right) \geq 0, \quad \frac{\partial G}{\partial \phi_{j+1}^{n+1}}=\lambda>0, \quad \frac{\partial G}{\partial \phi_{j-1}^{n+1}}=\lambda>0,
$$

where in the first inequality we have used the Assumption (2.2) and the induction assumption at $n$. The above result shows that $G$ is a monotone scheme, which yields

$$
\begin{aligned}
& (1+2 \lambda) \phi_{j}^{n+1}=G\left(\phi_{j}^{n}, \phi_{j+1}^{n+1}, \phi_{j-1}^{n+1}\right) \\
\leq & G\left(\gamma_{+}, \max _{j} \phi_{j}^{n+1}, \max _{j} \phi_{j}^{n+1}\right)=\gamma_{+}+2 \lambda \max _{j} \phi_{j}^{n+1},
\end{aligned}
$$

where we have used the assumption that $f\left(\gamma_{+}\right)=0$. As the above result is true for all $j$, we obtain

$$
(1+2 \lambda) \max _{j} \phi_{j}^{n+1} \leq \gamma_{+}+2 \lambda \max _{j} \phi_{j}^{n+1},
$$

which gives $\max _{j} \phi_{j}^{n+1} \leq \gamma_{+}$. Similarly, we have

$$
\begin{aligned}
& (1+2 \lambda) \phi_{j}^{n+1}=G\left(\phi_{j}^{n}, \phi_{j+1}^{n+1}, \phi_{j-1}^{n+1}\right) \\
\geq & G\left(\gamma_{-}, \min _{j} \phi_{j}^{n+1}, \min _{j} \phi_{j}^{n+1}\right)=\gamma_{-}+2 \lambda \min _{j} \phi_{j}^{n+1},
\end{aligned}
$$

where we have used the assumption that $f\left(\gamma_{-}\right)=0$. As the above result is true for all $j$, we obtain $\min _{j} \phi_{j}^{n+1} \geq \gamma_{-}$. This completes the proof of the theorem.

Before closing this section, we remark that the $L^{1}$-stability also holds for the AllenCahn equation (1.1a). More precisely, if the conditions (2.1) and (2.2) in Theorem 2.1 are satisfied, and also $f(0)=0$ with $\gamma_{-}<0<\gamma_{+}$, then the numerical solutions of (1.5) satisfy

$$
\left\|\phi^{n+1}\right\|_{1} \leq e^{L \Delta t}\left\|\phi^{n}\right\|_{1}
$$

where $L=-\min _{\gamma_{-} \leq u \leq \gamma_{+}} f^{\prime}(u)$, and

$$
\left\|\phi^{n}\right\|_{1}=\sum_{j=1}^{J}\left|\phi_{j}^{n}\right| \Delta x
$$


We briefly outline the proof of (2.7). It follows from the semi-implicit scheme (1.5) that

$$
(1+2 \lambda) \phi_{j}^{n+1}=\left(1-\Delta t f^{\prime}\left(\theta_{j}^{n}\right)\right) \phi_{j}^{n}+\lambda \phi_{j+1}^{n+1}+\lambda \phi_{j-1}^{n+1},
$$

where we have used the assumption $f(0)=0$ and $\theta_{j}^{n}$ is between 0 and $\phi_{j}^{n}$. Furthermore, it follows from (2.2) that the coefficient of $\phi_{j}^{n}$ is non-negative. Consequently, we have (2.9) that

$$
\begin{aligned}
(1+2 \lambda)\left|\phi_{j}^{n+1}\right| & \leq\left(1-\Delta t f^{\prime}\left(\theta_{j}^{n}\right)\right)\left|\phi_{j}^{n}\right|+\lambda\left|\phi_{j+1}^{n+1}\right|+\lambda\left|\phi_{j-1}^{n+1}\right| \\
& \leq(1+\Delta t L)\left|\phi_{j}^{n}\right|+\lambda\left|\phi_{j+1}^{n+1}\right|+\lambda\left|\phi_{j-1}^{n+1}\right| .
\end{aligned}
$$

The above result, together with the definition (2.8), leads to

$$
(1+2 \lambda)\left\|\phi^{n+1}\right\|_{1} \leq e^{L \Delta t}\left\|\phi^{n}\right\|_{1}+\lambda\left\|\phi^{n+1}\right\|_{1}+\lambda\left\|\phi^{n+1}\right\|_{1},
$$

which leads to the desired estimate (2.7).

\section{Energy stability}

A numerical correspondence of the energy definition (1.2) is given below

$$
E_{h}\left(\phi^{n}\right)=\frac{\epsilon^{2}}{2} \sum_{j=1}^{J}\left(\nabla_{+} \phi_{j}^{n}\right)^{2} \Delta x+\sum_{j=1}^{J} F\left(\phi_{j}^{n}\right) \Delta x .
$$

Theorem 3.1. Consider the semi-implicit scheme (1.5) with periodic boundary conditions. If the conditions (2.1) and (2.2) in Theorem 2.1 are satisfied, then the numerical solutions of (1.5) satisfy

$$
E_{h}\left(\phi^{n+1}\right) \leq E_{h}\left(\phi^{n}\right)
$$

Proof. It follows from the definition of the discrete energy (3.1) that

$$
\begin{aligned}
& E_{h}\left(\phi^{n+1}\right)-E_{h}\left(\phi^{n}\right) \\
= & \frac{\epsilon^{2}}{2} \sum_{j=1}^{J}\left(\nabla_{+} \phi_{j}^{n+1}+\nabla_{+} \phi_{j}^{n}\right)\left(\nabla_{+} \phi_{j}^{n+1}-\nabla_{+} \phi_{j}^{n}\right) \Delta x \\
& \quad+\sum_{j=1}^{J}\left(F\left(\phi_{j}^{n+1}\right)-F\left(\phi_{j}^{n}\right)\right) \Delta x \\
= & : \epsilon^{2} I_{1}+I_{2} .
\end{aligned}
$$


We now estimate $I_{1}$ and $I_{2}$. Note that

$$
\begin{aligned}
I_{1} & =\frac{1}{2} \sum_{j=1}^{J} \nabla_{+}\left(\phi_{j}^{n+1}+\phi_{j}^{n}\right) \nabla_{+}\left(\phi_{j}^{n+1}-\phi_{j}^{n}\right) \Delta x \\
& =\sum_{j=1}^{J} \nabla_{+} \phi_{j}^{n+1} \nabla_{+}\left(\phi_{j}^{n+1}-\phi_{j}^{n}\right) \Delta x-\frac{1}{2} \sum_{j=1}^{J}\left(\nabla_{+}\left(\phi_{j}^{n+1}-\phi_{j}^{n}\right)\right)^{2} \Delta x \\
& \leq \sum_{j=1}^{J} \nabla_{+} \phi_{j}^{n+1} \nabla_{+}\left(\phi_{j}^{n+1}-\phi_{j}^{n}\right) \Delta x \\
& =-\sum_{j=1}^{J} \nabla_{+} \nabla_{-} \phi_{j}^{n+1}\left(\phi_{j}^{n+1}-\phi_{j}^{n}\right) \Delta x,
\end{aligned}
$$

where in the last step we have used the discrete integration by parts. Furthermore, using Taylor expansion gives

$$
F\left(\phi_{j}^{n+1}\right)=F\left(\phi_{j}^{n}\right)+f\left(\phi_{j}^{n}\right)\left(\phi_{j}^{n+1}-\phi_{j}^{n}\right)+\frac{1}{2} f^{\prime}\left(\theta_{j}^{n}\right)\left(\phi_{j}^{n+1}-\phi_{j}^{n}\right)^{2},
$$

where $\gamma_{-} \leq \theta_{j}^{n} \leq \gamma_{+}$, which yields

$$
I_{2}=\sum_{j=1}^{J}\left(f\left(\phi_{j}^{n}\right)\left(\phi_{j}^{n+1}-\phi_{j}^{n}\right)+\frac{1}{2} f^{\prime}\left(\theta_{j}^{n}\right)\left(\phi_{j}^{n+1}-\phi_{j}^{n}\right)^{2}\right) \Delta x .
$$

It follows from the (3.3), (3.4) and (3.6) that

$$
\begin{aligned}
& E_{h}\left(\phi^{n+1}\right)-E_{h}\left(\phi^{n}\right) \\
& \leq \sum_{j=1}^{J}\left[-\epsilon^{2} \nabla_{+} \nabla_{-} \phi_{j}^{n+1}\left(\phi_{j}^{n+1}-\phi_{j}^{n}\right)+f\left(\phi_{j}^{n}\right)\left(\phi_{j}^{n+1}-\phi_{j}^{n}\right)\right] \Delta x \\
& \quad+\sum_{j=1}^{J} \frac{1}{2} f^{\prime}\left(\theta_{j}^{n}\right)\left(\phi_{j}^{n+1}-\phi_{j}^{n}\right)^{2} \Delta x .
\end{aligned}
$$

It follows from the semi-implicit scheme (1.5) that

$$
\frac{\left(\phi_{j}^{n+1}-\phi_{j}^{n}\right)^{2}}{\Delta t}=\varepsilon^{2} \nabla_{+} \nabla_{-} \phi_{j}^{n+1}\left(\phi_{j}^{n+1}-\phi_{j}^{n}\right)-f\left(\phi_{j}^{n}\right)\left(\phi_{j}^{n+1}-\phi_{j}^{n}\right) .
$$

Combining the above two results gives

$$
\begin{aligned}
& E_{h}\left(\phi^{n+1}\right)-E_{h}\left(\phi^{n}\right) \\
\leq & \sum_{j=1}^{J} \frac{\Delta x}{\Delta t}\left(-1+\frac{1}{2} \Delta t f^{\prime}\left(\theta_{j}^{n}\right)\right)\left(\phi_{j}^{n+1}-\phi_{j}^{n}\right)^{2} \\
\leq & 0,
\end{aligned}
$$

where in the last step we have used the Assumption (2.2) and the maximum principle result (2.3). 


\section{Concluding remarks}

This paper extends the result of [11] which provides similar maximum principle and energy stability results for the polynomial double well potential $F$. In [11], numerical experiments also demonstrated that the semi-implicit (sometimes also called implicitexplicit) method is an effective scheme for solving the Allen-Cahn equations.

It is demonstrated in [1] that semi-implicit scheme is very effective for the GinzburgLandau equation and the Cahn-Hilliard equation. We may conjecture that the energy stability (3.2) also holds for the semi-implicit solutions of the Cahn-Hilliard equation. However, standard energy analysis used in this work may not be sufficient to establish such a result. It is expected some deeper analysis is needed to verify the conjecture.

We point out that it is possible to extend the present framework to handle the CahnHilliard equation with a logarithmic free energy. The key ingredient for establishing the energy stability with the present framework is the boundedness of numerical solutions. The solution boundedness is indeed true for the Cahn-Hilliard equation with a logarithmic free energy [3]; however, there has very few theoretical justification of the numerical counterpart. It is worth mentioning two relevant works in this direction. One is the work of Copetti and Elliott [4] who analyzed the implicit Euler scheme and obtained the uniform maximum bound of the numerical solutions; another one is due to Chen et al. [2] who studied the first-order and second-order partially implicit scheme and obtained the $L^{\infty}$-bound of the numerical solutions. Of course, more interesting and challenging issue is to analyze the energy stability of semi-implicit schemes for the Cahn-Hilliard equation with a logarithmic free energy.

\section{References}

[1] L. Q. Chen and J. Shen, Applications of semi-implicit Fourier-spectral method to phase-field equations, Comput. Phys. Commun., 108 (1998), 147-158.

[2] W.-B. Chen, C. Wang, X.-M. Wang and S. M. Wise, Positivity-preserving, energy stable numerical schemes for the Cahn-Hilliard equation with logarithmic potential, J. Comput. Phys. X, 3 (2019), 100031.

[3] L. Cherfils, A. Miranville and S. Zelik, The Cahn-Hilliard equation with logarithmic potentials, Milan J. Math., 79 (2011), 561-596.

[4] M. I. M. Copetti and C. M. Elliott, Numerical analysis of the Cahn-Hilliard equation with a logarithmic free energy, Numer. Math., 63 (1992), 39-65.

[5] Q. Du and R. Nicolaides, Numerical analysis of a continuum model of phase transition, SIAM J. Numer. Anal., 28(5) (1991), 1310-1322.

[6] D. J. Eyre, Unconditionally gradient stable time marching the Cahn-Hilliard equation, in J. W. Bullard, R. Kalia, M. Stoneham, and L. Q. Chen, editors, Computational and Mathematical Models of Microstructural Evolution, volume 53, pages 1686-1712, Warrendale, PA, USA, 1998, Materials Research Society.

[7] D. Gilbarg and N. Trudinger, Ellipltic Partial Differential Equations of Second Order, Springer, 2015. 
[8] H. Liao, T. Tang and T. Zhou, On energy stable, maximum-principle preserving, second order BDF scheme with variable steps for the Allen-Cahn equation, SIAM J. Numer. Anal., 58 (2020), pp. 2294-2314.

[9] J. Shen, J. Xu, and J. Yang, A new class of efficient and robust energy stable schemes for gradient flows, SIAM Rev., 61(3) (2019), 474-506.

[10] Tao Tang, On effective numerical methods for phase-field models, in Proceedings of the International Congress of Mathematicians (ICM 2018), pages 3669-3690, IMU, 2018.

[11] T. Tang and J. Yang, Implicit-explicit scheme for the Allen-Cahn equation preserves the maximum principle, J. Comput. Math., 34(5) (2016), 471-481.

[12] C.-J. Xu and T. Tang, Stability analysis of large time-stepping methods for epitaxial growth models, SIAM J. Numer. Anal., 44(4) (2006), 1759-1779.

[13] J.-C. Xu, Y. K. Li, S. N. Wu, and A. Bousquet, On the stability and accuracy of partially and fully implicit schemes for phase-field modeling, Comput. Methods Appl. Mech. Eng., 345 (2019), 826-853. 